\title{
Synthesis and properties of a dual responsive hydrogel by inverse microemulsion polymerization
}

\author{
TAO WAN ${ }^{\mathrm{a}, \mathrm{b}, *}$, MIN XU $^{\mathrm{b}}$, LIYI CHEN ${ }^{\mathrm{a}, *}$, DAQING WUc, WENZHONG CHENG ${ }^{\mathrm{b}}$, RUIXIANG \\ $\mathrm{LI}^{\mathrm{b}}$ and CHUZHANG ZOU

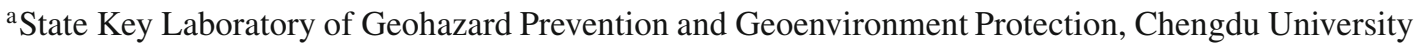 \\ of Technology, Chengdu 610059, China \\ ${ }^{\mathrm{b}}$ Mineral Resources Chemistry Key Laboratory of Sichuan Higher Education Institutions, Chengdu University \\ of Technology, Chengdu 610059, China \\ ${ }^{\mathrm{c}}$ PetroChina Sichuan Petrochemical Co., Ltd., Chengdu 611930, China \\ e-mail: wantaos@126.com; cly@cdut.edu.cn
}

MS received 4 July 2014; revised 24 August 2014; accepted 30 August 2014

\begin{abstract}
A novel dual responsive hydrogel was synthesized by inverse microemulsion polymerization, using itaconic acid as $\mathrm{pH}$-responsive monomer and $\mathrm{N}$ - isopropylacrylamide as thermo-responsive monomer. $\mathrm{pH}$ - and temperature-sensitivity and dynamic viscoelasticity behaviour of the dual responsive hydrogels were investigated. Dual responsive hydrogels showed remarkable $\mathrm{pH}$ - and temperature-sensitivity and enhanced viscoelastic behaviour under high stress. Temperature-induced shrinkage range of the dual responsive hydrogels was higher and broader than that of the conventional poly(N-isopropylacrylamide) hydrogel. FTIR revealed the structure of dual responsive hydrogels. The as-synthesized regular and sphere-like hydrogel particles had the average particle size of $49 \mathrm{~nm}$ in the range of $30-78 \mathrm{~nm}$.
\end{abstract}

Keywords. Hydrogel; microemulsion polymerization; sensitivity; N-isopropylacrylamide.

\section{Introduction}

Intelligent hydrogels undergoing significant changes in their physicochemical properties in response to minor changes in external stimuli, such as temperature, ${ }^{1,2}$ $\mathrm{pH},{ }^{3}$ solvent composition, ${ }^{4,5}$ light, ${ }^{6}$ magnetic field, ${ }^{7}$ and ionic strength, ${ }^{8}$ have attracted much attention because they can serve as functional materials with potential applications in the areas of drug delivery, ${ }^{9}$ microlenses, ${ }^{10}$ sensors ${ }^{11}$ and artificial organ, ${ }^{12}$ etc.

Dual responsive hydrogels that are both temperatureand $\mathrm{pH}$-sensitive are the most widely investigated because these two stimuli are the most commonly used external stimuli. ${ }^{13}$ Poly(N-isopropylacrylamide) (PNIPAM) is the most popular temperature-responsive hydrogel due to its volume phase transition temperature (around $32^{\circ} \mathrm{C}$ ) close to normal body temperature. PNIPAM has been extensively studied due to its thermal sensitive property and can react with acrylic acid or other polymers to design new multifunctional materials. ${ }^{14-19}$

On the basis of our previous research on superabsorbent hydrogels and microemulsion polymerization, ${ }^{20-31}$ a novel dual responsive hydrogel with

*For correspondence average particle size of $49 \mathrm{~nm}$ was prepared by inverse microemulsion polymerization, using itaconic acid as $\mathrm{pH}$-responsive monomer, $\mathrm{N}$-isopropylacrylamide as thermo-responsive monomer and acrylamide as the non-ionic hydrophilic monomer. The dual responsive hydrogel showed good $\mathrm{pH}$ - and temperature-sensitivity and water swellability.

\section{Experimental}

\subsection{Materials}

Itaconic acid (IA) was purified by recrystallization. Acrylamide (AM), N-isopropyl acrylamide (NIPAM) and N-methylene-bis- acrylamide (MBA) were purified by recrystallization. Ammonium persulfate (APS), sodium bisulfite (SBS), sorbitan mono-oleate (Span80), polyoxyethylene sorbitan monooleate (Tween 80), cyclohexane were used without further purification.

\subsection{Synthesis of dual responsive hydrogels}

Dual responsive hydrogel was synthesized according to the following steps. Typically, $3 \mathrm{~g}$ IA was dissolved 
in $10 \mathrm{~mL}$ distilled water and neutralized with $20 \%$ sodium hydroxide solution in an ice bath, and then $4 \mathrm{~g}$ AM, $2 \mathrm{~g}$ NIPAM and $0.036 \mathrm{~g}$ MBA were added to the above monomer solution and the mixed solution was stirred at room temperature for $30 \mathrm{~min}$. Subsequently the solution was added dropwise to the inverse microemulsions formed by $100 \mathrm{~g}$ cyclohexane, $6 \mathrm{~g}$ Span 80 , and $4 \mathrm{~g}$ Tween 80 . The water bath was heated slowly to $45-55^{\circ} \mathrm{C}$ with mild stirring after redox initiator APS $(55 \mathrm{mg})$ and SBS $(15 \mathrm{mg})$ were introduced to the above inverse microemulsions. After $3-5 \mathrm{~h}$ of the reaction, the resulting product was washed several times with ethanol and acetone, respectively, dried at $60^{\circ} \mathrm{C}$ to a constant weight, and then milled and screened.

\subsection{Water absorbency of dual responsive hydrogels using filtration method}

Approximately $100 \mathrm{mg}$ of dried, milled and sifted hydrogels were dispersed in $100 \mathrm{~mL}$ of deionized water for $300 \mathrm{~min}$. Then, excess water was allowed to drain through a 300 mesh wire gauze. The weight of the hydrogels containing absorbed water was measured after draining for $30 \mathrm{~min}$, and water absorbency was calculated according to the following equation:

$$
\text { Absorbency }(\mathrm{g} / \mathrm{g})=\left(W_{2}-W_{1}\right) / W_{1}
$$

where $W_{1}$ and $W_{2}$ are the weight of the dry and swollen hydrogels, respectively.

\subsection{Dynamic viscoelasticity measurements of the dual responsive hydrogels}

Dynamic viscoelasticity measurements were performed with a Rheometric Fluids RFR 7800 rheometer. The applied frequency $(\omega)$ was changed from 0.1 to $10 \mathrm{rad} / \mathrm{s}$. The storage $\left(\mathrm{G}^{\prime}\right)$ and loss $\left(\mathrm{G}^{\prime \prime}\right)$ moduli were obtained from oscillatory measurements with stress of $50 \mathrm{~Pa}$, $100 \mathrm{~Pa}$ and $200 \mathrm{~Pa}$, respectively.

\subsection{Characterization of the dual responsive hydrogels}

The micrographs of dual responsive hydrogels were taken using TEM (Tecnai G2 F20S-TWIN, FEI). Before TEM observation, inverse microemulsion samples were diluted with cyclohexane, stained with phosphotungstic acid, and then dipped onto the copper grid and allowed to evaporate at room temperature. FT-IR spectrum was carried out on a Perkin-Elmer 1750 spectrophotometer, equipped with an Epson Endeavour II data station. The dried samples were crushed with $\mathrm{KBr}$ to get pellets.

\section{Results and Discussion}

\subsection{Morphology and structure of the as-synthesized dual responsive hydrogels}

Morphology of the as-synthesized dual responsive hydrogels was characterized by TEM and the result was shown in figure 1 . The hydrogel particles are regular and spherical-like in shape, and have the average particle size of $49 \mathrm{~nm}$ in the range of $30-78 \mathrm{~nm}$, indicating that dual responsive hydrogels with nanoscale size, have been successfully synthesized by microemulsion polymerization.

FTIR spectrum of dual responsive hydrogels was demonstrated in figure 2. The peak at $3445 \mathrm{~cm}^{-1}$ is attributed to $\mathrm{OH}$ and $\mathrm{NH}$ stretching vibrations. The peaks at 2920 and $2854 \mathrm{~cm}^{-1}$ are ascribed to the $\mathrm{CH}_{2}$ asymmetric and $\mathrm{CH}_{2}$ symmetric stretch, respectively. ${ }^{32}$ The absorption bands at $1720 \mathrm{~cm}^{-1}$ are assigned to carboxylic acid. ${ }^{33}$ The bands at $1660 \mathrm{~cm}^{-1}$ and $1550 \mathrm{~cm}^{-1}$ represent amide group stretching. ${ }^{34}$ The absorption peak at $1385 \mathrm{~cm}^{-1}$ is attributed to isopropyl group. The peak at $1050 \mathrm{~cm}^{-1}$ arises from C- O stretching

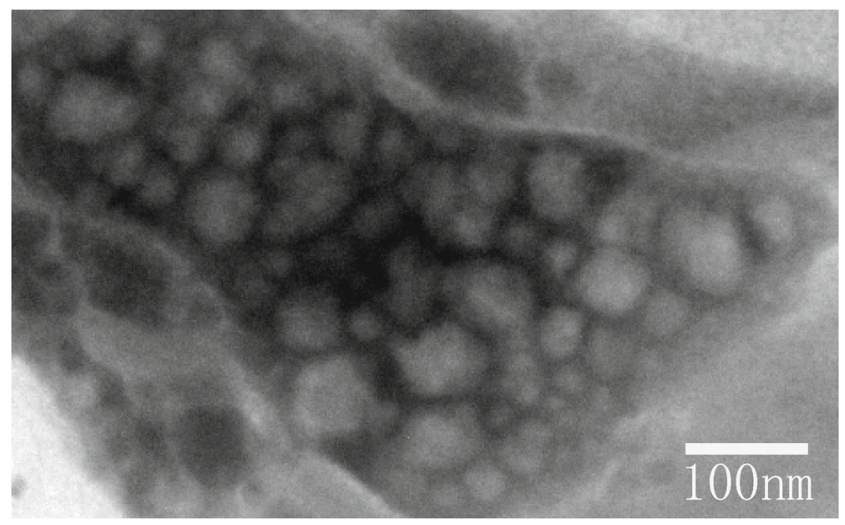

Figure 1. TEM image of the dual responsive hydrogels synthesized at IA/ NIPAM mass ratio of 70:30.

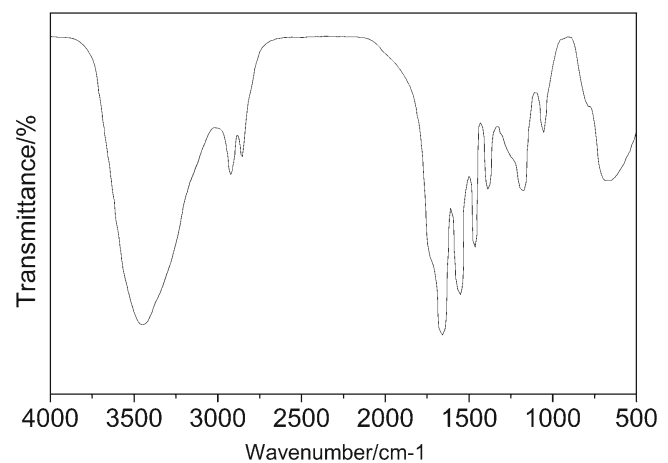

Figure 2. FTIR spectrum of the dual responsive hydrogels synthesized by inverse microemulsion polymerization. 
vibration. All the above results revealed the formation of dual responsive poly (NIPAM/IA/AM) hydrogels.

\subsection{Temperature-sensitivity of the dual responsive hydrogels}

Temperature-dependent swelling behaviours of the dual responsive hydrogels were investigated with different temperatures and IA/NIPAM mass ratio, and the results were shown in figure 3. Swelling capacities of all hydrogels decreased with temperature increasing from 30 to $70^{\circ} \mathrm{C}$, and the swelling behaviour of hydrogels around $30-50^{\circ} \mathrm{C}$ displayed an increasing sharp decrease with decreasing IA/NIPAM mass ratio, indicating an increased temperature sensitivity of the dual responsive hydrogels with decreasing IA/NIPAM mass ratio. The NIPAM moieties become dehydrated (and effectively hydrophobic in nature) when heated above their LCST (i.e., $\mathrm{T}>32^{\circ} \mathrm{C}$ ), ${ }^{35}$ associate and form thermoreversible aggregates, decreasing Flory-Huggins interaction parameter $\chi$ between the solvent and the hydrogel, thus inducing changes in the osmotic pressure. This results in the shrinkage of the polymeric network, release of the absorbed water and decrease of swelling capacity. It is of interest to note that the shrinkage temperature range (around $30-50^{\circ} \mathrm{C}$ ) of the dual responsive hydrogels was higher and broader than that of the conventional PNIPAM hydrogel (around $32^{\circ} \mathrm{C}$ ). This might be ascribed to the fact that LCST can be enhanced by the copolymerization of NIPAM with hydrophilic or ionized monomersm. ${ }^{36}$

\section{$3.3 p H$-sensitivity of the dual responsive hydrogels}

$\mathrm{pH}$-dependent swelling behaviours of the dual responsive hydrogels were investigated with changes in $\mathrm{pH}$ and IA/NIPAM mass ratio, as shown in figure 4 . Water

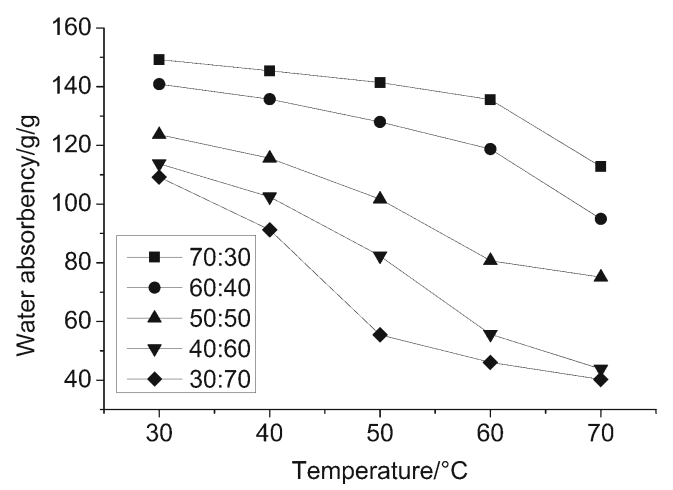

Figure 3. Temperature sensitivity of the dual responsive hydrogels synthesized at different IA/ NIPAM mass ratio.

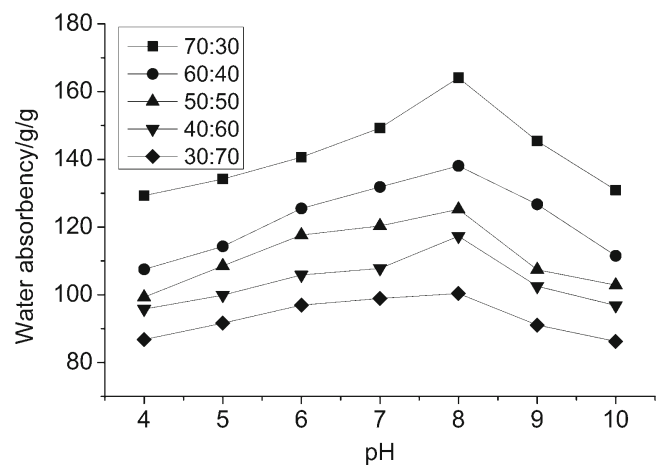

Figure 4. $\mathrm{pH}$ sensitivity of the dual responsive hydrogels synthesized at different IA/ NIPAM mass ratio.

absorbency increased as $\mathrm{pH}$ increased to 8 , and then decreased with further increase of $\mathrm{pH}$ value. In addition, $\mathrm{pH}$-sensitivity of the dual responsive hydrogels increased with increasing IA/NIPAM mass ratio. At a low $\mathrm{pH}$ region, most carboxylic acid groups in dual responsive hydrogels are in the form of $\mathrm{COOH}$. As the $\mathrm{pH}$ of the medium increases, the carboxylic acid groups become ionized, and the resulting electrostatic repulsion in the network causes the hydrogels to swell. Besides, the amount of $\mathrm{Na}^{+}$ions rises with increasing $\mathrm{pH}$ value, leading to the enhancement of the ionic strength in the polymeric network and thereby the improvement of osmotic pressure difference and water absorption capacity. However, more $\mathrm{Na}^{+}$ions will introduce into the hydrogels with the $\mathrm{pH}$ value exceeding 8 , reducing the repulsion by screening the negative charges of $\mathrm{COO}^{-}$groups ${ }^{37}$ As a result, both the osmotic pressure of the gel phases and the chain relaxation process within the network are reduced and consequently the water absorption capacity decreases.

\subsection{Dynamic rheology of the dual responsive hydrogels}

Dynamic rheology behaviours of the dual responsive hydrogels were shown in figure 5. Both storage moduli $G^{\prime}$ and loss moduli $G^{\prime \prime}$ of the dual responsive hydrogels changed little with increasing oscillation frequency under stress of $50 \mathrm{~Pa}, 100 \mathrm{~Pa}$ and $200 \mathrm{~Pa}$, respectively. Solid-like behaviours (storage moduli $G^{\prime}>$ loss moduli $G^{\prime \prime}$ ) were observed due to the three-dimensional network structure. With the increase of stress, $G^{\prime}$ decreased, whereas $G^{\prime \prime}$ gradually increased, indicating enhanced viscoelastic behaviours of the dual responsive hydrogels under high stress. Polymeric chains of the hydrogels are more liable to stretch, twist and relax under high stress, resulting in an increased liquid-like behaviour and decreased solidlike behaviour. Therefore the dual responsive hydrogels 

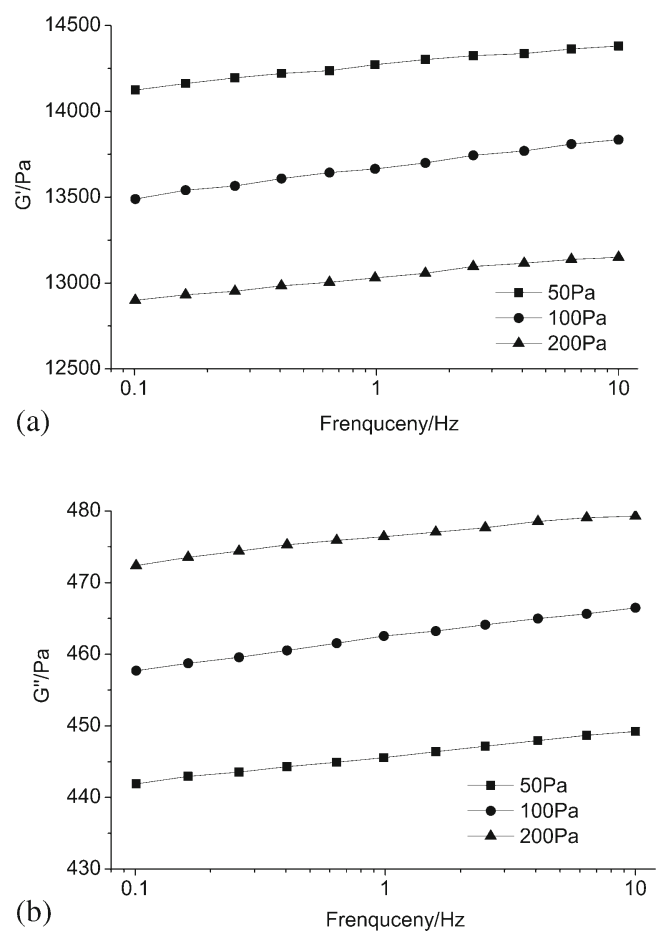

Figure 5. Variation of the storage modulus $\mathrm{G}^{\prime}$ (a) and the loss modulus $G^{\prime \prime}$ (b) with the oscillation frequency for the dual responsive hydrogels at different stress.

show remarkable viscoelastic behaviours under high stress.

\section{Conclusions}

For the first time, a new dual responsive hydrogel was synthesized by facile inverse microemulsion polymerization, using IA as $\mathrm{pH}$-responsive monomer and NIPAM as thermo-responsive monomer. $\mathrm{pH}$-sensitivity and temperature-sensitivity of the dual responsive hydrogels increased and decreased with increasing IA/NIPAM mass ratio, respectively. Temperatureinduced shrinkage range (around $30-50^{\circ} \mathrm{C}$ ) of the dual responsive hydrogels was higher and broader than that of the conventional PNIPAM hydrogel (around $32^{\circ} \mathrm{C}$ ). Dual responsive hydrogels show enhanced viscoelastic behaviour under high stress. FTIR demonstrated the formation of dual responsive poly(NIPAM/IA/AM) hydrogels. TEM indicated that the as-synthesized hydrogel particles were regular and sphere-like in shape and had average particle size of $49 \mathrm{~nm}$ in the range of $30-78 \mathrm{~nm}$.

\section{Acknowledgements}

The authors are grateful to the support of PetroChina Innovation Foundation, China (2012D-5006-0212),
Sichuan Provincial Science \& Technology Pillar Program, China (2013GZ0149, 2014GZX0010), Opening fund of State Key Laboratory of Geohazard Prevention and Geoenvironment Protection, Chengdu University of Technology, China (SKLGP2012K004) and the Sichuan Youth Science and Technology Innovation Research Team Funding Scheme, China (2013TD0005).

\section{References}

1. Kiler J, Scranton A B and Peppas N A 1990 Macromolecules 234944

2. Xue F F, Yuan D D, Sahasrabudhe A, Biswas S, Wang P, Tang X Y, Chen D Y, Yuan R X and Roy S 2012 New J. Chem. 362541

3. Chiu H C, Lin Y F and Hung S H 2002 Macromolecules 355235

4. Roy S G, Haldar U and De P 2014 ACS Appl. Mater. Interfaces 64233

5. Kokufata E, Zhang Y Q and Tanaka T 1991 Nature 351 302

6. Mamada A, Tanaka T, Kungwatchakun D and Irie M 1990 Macromolecules 231517

7. Xulu P M, Filipcsei G and Zrinyi M 2000 Macromolecules $\mathbf{3 3} 1716$

8. Park T G and Hoffman A S 1993 Macromolecules 26 5045

9. Ward M A and Georgiou T K 2011 Polymers 31215

10. Dong L and Jiang H 2007 Soft Matter. 31223

11. Hoare T and Pelton R 2007 Macromolecules 40670

12. Shiino D, Murata Y, Kataoka K, Koyama Y, Yokoyama M, Okano T and Sakurai Y 1994 Biomaterials 15121

13. Zhao C W, Zhuang X L, He P, Xiao C S, He C L, Sun J R, Chen X S, Jing X B 2009 Polymer 504308

14. Zhang X, Yang Y, Wang F and Chung T 2002 Langmuir. 182013

15. Zhang J, Xu S and Kumacheva E 2004 J. Am. Chem. Soc. 1267908

16. Muratalin M and Luckham P F 2013 J. Colloid. Interf. Sci. 3961

17. Stile R A, Burghardt W R and Healy K E 1999 Macromolecules $\mathbf{3 2} 7370$

18. Nishioa T, Ayanoa E, Suzuki Y, Kanazawaa H and Okanob T 2011 J. Chromatogr. A 12182079

19. Burova T V, Grinberg N V, Lozinskii V I, Moiseeva S P, Kotel'nikov G V, Grinberg V Y and Khokhlov A R 2010 Polym. Sci. Ser. A 52356

20. Wan T, Xiong L, Huang R Q, Zhao Q H, Tan X M, Qin L L and Hu J Y 2014 Polym. Bull. 71371

21. Wan T, Huang R Q, Zhao Q H, Xiong L, Luo L, Zhang H B and Cai G J 2014 J. Compos. Mater. 482341

22. Wan T, Huang R Q, Zhao Q H, Xiong L, Luo L, Tan X M and Cai G J 2013 J. Appl. Polym. Sci. 130698

23. Wan T, Huang R Q, Zhao Q H, Xiong L, Qin L L, Tan X M and Cai G J 2013 J. Appl. Polym. Sci. 1303404

24. Wan T, Yao J, Sun Z S, Wang L and Wang J $2011 \mathrm{~J}$. Petrol. Sci. Eng. $\mathbf{7 8} 334$

25. Wan T, Zang T S, Wang Y C, Zhang R and Sun X C 2010 Polym. Bull. 65565 
26. Wan T, Wu C, Ma X L, Yao J and Lu K 2009 Polym. Bull. 62801

27. Wan T, Yao J and Ma X L 2008 J. Appl. Polym. Sci. 110 3859

28. Wan T, Wang L, Yao J, Ma X L, Yin Q S and Zang T S 2008 Polym. Bull. 60431

29. Wan T, Hu Z W, Ma X L, Yao J and Lu K 2008 Prog. Org. Coat. 62219

30. Wan T, Wang X Q, Yuan Y and He W Q 2006 Polym. Int. 551413

31. Wan T, Wang X Q, Yuan Y and He W Q 2006 J. Appl. Polym. Sci. 1022875
32. Mendelsohn R, Flach C R and Moore D J 2006 BBABiomembranes. 1758923

33. Chen G, Zhang Y and Zhou X 2006 Appl. Surf. Sci. 253 1107

34. Krishnan M and Flanagan D R 2000 J. Control. Release. 69273

35. Zhao $\mathrm{C} \mathrm{W}$, Gao $\mathrm{X} \mathrm{Y}$, He $\mathrm{P}$, Xiao C S, Zhuang X L and Chen X S 2011 Colloid. Polym. Sci. 289 447

36. Gil E S and Hudson S M 2004 Prog. Polym. Sci. 291173

37. Ma J H, Xu Y J, Zhang Q S, Zha L S and Liang B R 2007 Colloid Polym. Sci. 285479 